motor function, feeding rejection and oral hypersensitivity problems. Maternal depression was assessed by the Edinburgh Postpartum Depression Scale. Prenatal, perinatal and postnatal risk factors were recorded from the family and the hospital records. A development evaluation was performed with the Denver II Development Screening Test (DDST-II).

Results Demographic characteristics are summarized in table-1. Twenty-six of the patients $(44.8 \%)$ had feeding rejection, 9 (15.5\%) had problems with oral-motor function, 2 (3.4\%) had oral hypersensitivity problems and 8 (13.8\%) had multiple feeding problems. No relation was found between birth weight and feeding problems. Parameters that are significantly associated with feeding problems in patients are shown in table-2. In the developmental evaluation, $14(24.1 \%)$ of the patients were found to be abnormal in DDST-II and 10 of these patients $(71.4 \%)$ had feeding problems (p: 0.066).

\begin{tabular}{ll} 
Abstract P132 Table $\mathbf{1}$ \\
\hline Table-1. Demographic characteristics \\
\hline Gender (F/M) & $23 / 35$ \\
Age (months \pm SD) & $20.6 \pm 2.97$ \\
Gestational age (weeks \pm SD) & $32.3 \pm 2.9$ \\
Birth weight (gram \pm SD) & $1731.1 \pm 662.2$ \\
Birth weight <1500 gr (n (\%)) & $27(46.6 \%)$ \\
Small for gestational age $(\mathrm{n}(\%))$ & $7(12.1 \%)$ \\
Resusitation in the delivery room (n (\%)) & $27(46.6 \%)$ \\
Apgar score 5th min $<7(\mathrm{n}(\%))$ & $10(17.2 \%)$ \\
Weight (gram \pm SD) & $11100 \pm 1990$ \\
Weight <3 percentiles (n (\%)) & $7(12.1 \%)$ \\
Maternal depression $(\mathrm{n}(\%))$ & $13(22.4 \%)$ \\
\hline
\end{tabular}

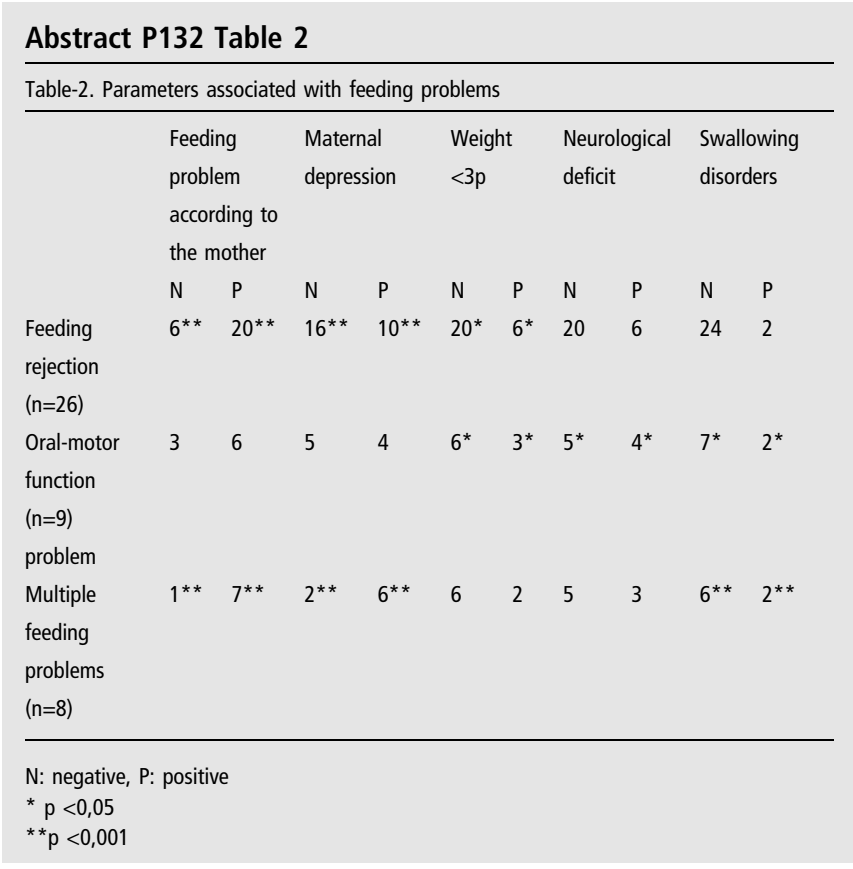

Conclusion Since the presence of feeding problems affect weight gain and feeding behaviors, it is important to identify and diagnose early risk factors. In particular, mother's concerns about feeding problems of the child should be questioned and the mother should be evaluated for depression.

\section{P133 THE IMPACT OF DIFFERENT DIETARY PATTERNS DURING PREGNANCY AND IN THE FIRST YEAR OF LIFE ON THE CHILDREN LENGTH-WEIGHT GROWTH UP TO 12 MONTHS OF AGE}

${ }^{1}$ Pietro Ferrara*, ${ }^{2}$ Federica Sandullo, ${ }^{2}$ Federica Di Ruscio, ${ }^{2}$ Giulia Franceschini, ${ }^{2}$ Roberta Autuori, ${ }^{2}$ Alessandro Di Lucia, ${ }^{3}$ Benedetta Peronti, ${ }^{4}$ Valeria Blasi, ${ }^{5}$ Sabina Bietolini, ${ }^{1}$ Antonio Chiaretti, ${ }^{6}$ Massimo Pettoello-Mantovani. ${ }^{1}$ Institute of Pediatrics, Catholic University Medical School, Rome, Italy; ${ }^{2}$ Service of Pediatrics, Campus Bio-Medico University, Rome, Italy; ${ }^{3}$ Unit of Food Science and Human Nutrition, Campus Bio-Medico University, Rome, Italy; ${ }^{4}$ Food Sciences and Human Nutrition Unit, Fondazione Policlinico Universitario Agostino Gemelli, Rome, Italy; ${ }^{5}$ Nutritionist Biologist, Rome, Italy; ${ }^{6}$ Department of Pediatrics, Scientific Institute Casa Sollievo della Sofferenza, University of Foggia, Foggia, Italy

\subsection{6/archdischild-2019-epa.488}

Objectives The aim of the present study is to compare the length-weight growth up to 12 months of age of three groups of children according to the dietary pattern followed by the pregnant mothers and by the infants during the first year of the life.

Design This is a retrospective study

Setting A questionnaire of 26 multiple-choise questions was drafted and electronically sent to the participants. From the questionnaire the following anthropometric data of the children were obtained: weight, length and head circumference, at birth, at 6 months and at 12 months; the corresponding BMI were calculated from them. Then, the percentiles of growth of all the parameters were calculated. Data were expressed as the arithmetic mean, standard deviation, median and interquartile range and a statistical analysis through ANOVA was conducted, with analysis post-hoc in accordance with the Bonferroni method. Values of $P$ less than 0,05 were considered significant. This study was conducted in accordance with the regulatory standards of Good Clinical Practice and the Declaration of Helsinki.

Patients 55 mothers and their 63 children were enrolled from the Pediatric Service of the Campus Bio-Medico University Hospital of Rome, between September 2017 and May 2018. The participants were divided into three groups based on the food patterns followed: 21 mothers and 21 children vegan, 19 mothers and 21 children lacto-ovo-/lacto-vegetarian, and 15 mothers and 21 children omnivore.

Main outcome measures Comparison of the anthropometric parameters of three groups of children according to the dietary pattern followed by the pregnant mothers and by the infants during the first year of the life.

Results From the statistical analysis, statistically significant differences emerged between the vegan group (lower values) and the omnivorous one regarding the children weight at birth, at 6 months and at 12 months, both when expressed in grams and when expressed as percentile of growth, the children length at 12 months expressed in percentile of growth and the children BMI at 6 months. No statistically significant differences emerged for the other parameters analysed between the omnivorous group and the vegan one and for no parameter between the vegan group and the lacto-ovo-/lacto-vegetarian one and between the latter and the omnivorous group.

Conclusion The limited number of participants does not allow us to draw unequivocal conclusions, but, despite the differences between the groups examined, we found during the first year of life a physiological and harmonic growth of all children. 